\title{
The Effects of Nefazodone on Sleep Architecture in Depression
}

Roseanne Armitage, Ph.D., A. John Rush, M.D., Madhukar Trivedi, M.D., John Cain, M.D., and Howard P. Roffwarg, M.D.

A polysomnographic study was conducted on 10 outpatients with major depression at baseline and during 4 to 8 weeks of open-trial treatment with nefazodone (400 to $600 \mathrm{mg} /$ day). All 10 patients were treatment responders as evidenced by at least $50 \%$ reduction from baseline scores on the Hamilton Depression Rating Scale. Nefazodone was associated with significantly decreased wake and movement time and increased minutes and percentage of stage 2 sleep at the expense of light stage 1 sleep. Nefazodone did not increase rapid-eye-movement
(REM) latency and it did not suppress REM sleep. In fact, a trend toward increased REM in the second REM period was observed, although decreased REM in the third REM period was also noted.

In summary, nefazodone, an effective antidepressant, decreases arousals and wakefulness during sleep and reduces light non-REM sleep. This agent does not appear to suppress REM sleep or prolong REM latency in patients who respond to treatment.

[Neuropsychopharmacology 10:123-127, 1994]
KEY WORDS: Electroencephalographic sleep; Depression; Nefazodone; 5- $\mathrm{HT}_{2}$; Antidepressants

Rapid-eye-movement (REM) sleep suppression has been proposed as a mechanism of action of antidepressant medications (Vogel et al. 1972; Vogel 1983). In support of this, Kupfer et al. (1981) have shown that a drug-induced increase in REM latency predicted antidepressant response, and under some circumstances, drug-induced REM suppression predicted symptom improvement (Kupfer et al. 1981). With the exception of trimipramine, a sedating tricyclic (Ware et al. 1989), most antidepressant drugs of this class, and also monoamine oxidase inhibitors (Kay et al. 1976), inhibit REM sleep (Vogel et al. 1990). Nefazodone, a phenylpiperazine agent, is a serotonin-2 (5- $\left.\mathrm{HT}_{2}\right)$ receptor antagonist and a selective 5-HT reuptake inhibitor (Eison et al. 1990). It is chemically distinct from tricyclic antidepressants and from first- and second-generation 5-HT reuptake inhib-

From the University of Texas Southwestern Medical Center, Dallas, Texas.

Address correspondence to: Roseanne Armitage, Ph.D., Department of Psychiatry, The University of Texas Southwestern Medical Center at Dallas, 5323 Harry Hines Blvd., Dallas, Texas, 75235-9070.

Received June 25, 1993; revised November 24, 1993; accepted November 30, 1993. itors. Recent clinical trials indicate that nefazodone is an effective antidepressant drug (Feighner et al. 1989; Fontaine et al. 1991) that may increase REM sleep in normal subjects (Ware et al. 1991; Sharpley et al. 1992).

Major depression has been linked to abnormalities of 5-HT neurotransmission (Rush et al. 1991). Research has shown that administration of agents that block synthesis of 5-HT as well as ingestion of compounds that deplete tryptophan results in increases in depressive symptoms (Delgado et al. 1991). Inasmuch as 5-HT is implicated in the onset of sleep (Jones 1991) and perhaps in the regulation of both REM and non-REM (NREM) sleep (Siegel 1990), nefazodone and other compounds that alter 5-HT metabolism might be expected to alter sleep architecture in patients suffering from major depression. The purpose of this pilot study was to investigate the effects of nefazodone on sleep in depression.

\section{METHODS}

\section{Subjects}

Subjects, 18 years of age or older, who met DSM-III-R criteria for nonpsychotic, major depression, but who 
were otherwise physically healthy, and who provided written informed consent were included. Excluded were those individuals currently engaged in shiftwork and those with independent sleep disorders (e.g., narcolepsy, apnea, bruxism, myoclonus) as established by history or polysomnogram. In addition, other current axis I disorders, current medical conditions, or psychoactive substance use within 12 months prior to baseline measurements caused subjects to be excluded. Women were excluded if they were pregnant or lactating or were not using an adequate method of contraception.

Diagnoses were made based on the structured clinical interview for DSM-III-R (Spitzer et al. 1986). A 17item Hamilton Depression Rating Scale(HDRS) (Hamilton 1960) was also used, and a detailed personal and family history was obtained at the time of clinical interview. Ten outpatients with major depression (five men, five women), averaging $42.9(\mathrm{SD}=9.9)$ years of age, participated in the study. All patients were symptomatic (HDRS $=20.7 ; \mathrm{SD}=4.3$ ) at the time of study and had an average onset of depression at age 27.7 (SD = 11.7) years. Seven of the 10 patients had recurrent major depression (three patients had more than five episodes), whereas three were experiencing a first episode of depression. One patient was clearly endogenous, five were probable endogenous, and four were nonendogenous by Research Diagnostic Criteria (Spitzer et al. 1986). None had melancholic features by DSM-III-R. All patients knew they would be treated with nefazodone.

\section{Procedure}

Each subject maintained individualized, regular bed and rise times for at least the 5 days prior to polysomnographic (PSG) study, as assessed by home diary. The subjects spent two consecutive baseline nights in the University of Texas Southwestern Medical Center Sleep Study Unit. Patients returned to the laboratory for 2 additional nights after no less than 4 weeks of nefazodone treatment. Electroencephalographic (EEG) data were collected from left (C3) and right (C4) central electrodes with a linked-ear reference. Monopolar, left and right electrooculograms, and bipolar chin-cheek electromyograms were also recorded. A full electrode montage, used on the first night in the sleep laboratory, included leg, chest, and abdomenleads, and a nasal-oral thermistor to rule out independent sleep disorders. All electrophysiologic signals were recorded on GRASS P-511 AC amplifiers and displayed on a paperless polygraph system. An amplifier sensitivity of 5 was used for $\operatorname{EEG~}(50 \mu \mathrm{V}, 0.5$-second duration calibration), with half-amp low- and high-bandpass filters set at 0.3 and $30 \mathrm{~Hz}$. A $60-\mathrm{Hz}$ notch filter attenuated electrical noise. Interelectrode impedances were maintained below 2
Khom. Amplifiers were calibrated before and after each night's sleep. As is standard procedure in our laboratory, EEG amplifiers were counterbalanced between the hemispheres, across subjects, and between nights to rule out amplifier artifact as a contributing source to interhemispheric differences (Armitage et al. 1989) routinely evaluated in computer-analyzed EEG frequencies, but not reported here.

\section{Dosing Procedure}

All patients began treatment by taking $100 \mathrm{mg} /$ day of nefazodone at bedtime for 3 days, followed by $100 \mathrm{mg}$ bid. Dose was then increased to $100 \mathrm{mg}$ in the morning and $200 \mathrm{mg}$ in the evening for 3 additional days. By the end of the 2nd week of treatment, the dose was $200 \mathrm{mg}$ in the morning and $200 \mathrm{mg}$ in the evening and was increased to a total daily dose of 500 to $600 \mathrm{mg} /$ day if deemed necessary by the attending physician. Patients received an average dose of $520 \mathrm{mg} /$ day $(S D=79)$ of nefazodone (range 400 to $600 \mathrm{mg} /$ day) during the 7 days preceding the sleep study. The average length of drug treatment following stabilization of dose was 48.7 (SD = 7.1) days. The mean HDRS score at the time of the second sleep study was $8.2(\mathrm{SD}=3.2)$, more than a $60 \%$ reduction from the pretreatment scores.

Sleep EEG records were scored from C3 according to standard Rechtschaffen and Kales (1968) criteria by technicians trained at better than $90 \%$ agreement on an epoch-by-epoch basis. A number of sleep variables were derived from the sleep-stage score data. Total time in bed was computed as the total time from lights-out to wake-up time. Sleep onset latency was defined as the time elapsed before the first epoch of sleep in a 10 minute segment containing no more than 2 minutes of wakefulness or the time in minutes to the first halfminute of REM sleep, whichever was sooner (Rush et al. 1989). Total sleep period (TSP) was defined as the length of time from sleep onset to wake up. Sleep efficiency was computed as the ratio of all sleep time to total time in bed. Rapid-eye-movement latency was equal to the number of minutes, including wake and movement time, from sleep onset to the first epoch of REM sleep (irrespective of REM period duration). The length or duration of REM period was defined as the first to the last half-minute REM epoch, in an interval of REM sleep unbroken by more than 30 minutes of NREM or awake and movement time (REMDUR1, REMDUR2, and REMDUR3). The number of minutes of net REM in each REM period was also computed (REMIN1, REMIN2, and REMIN3). Minutes and percentages of each sleep stage and of awake and movement time were computed in relation to TSP. Arousals were defined as the total number of awake episodes of 0.5 minutes or longer duration in TSP. 


\section{RESULTS}

Eight patients remitted with treatment (HDRS $\leqslant 10$ ). The remaining two patients also responded to treatment as evidenced by greater than $50 \%$ reduction in the HDRS scores (final HDRS scores: 11 and 13). All $10 \mathrm{Ss}$ were included in the statistical analyses. The data were coded for night-in-study and treatment level (baseline [drug-free] $T_{1}$ versus [on nefazodone] $T_{2}$ ) and repeated-measures analyses of variance were computed.

Table 1 presents the means and standard deviations of all sleep measures by treatment condition. Although the convention is to report significance at $\alpha=.05$, this study is based on a small sample size. In actuality, effect sizes can be quite large but can fail to reach significance due to low statistical power. As such, trends are indicated at probabilities below .10 (Stevens 1986). As no evidence of a statistically significant night effect was found, the data were averaged and analyses were recomputed based on the means of nights 1 and 2 . To be compatible with existing published data, both min-
Table 2. Analysis of Variance Summary*

\begin{tabular}{lrcc}
\hline Variable & $\boldsymbol{F}$ & $\boldsymbol{d f}$ & $\boldsymbol{p}$ \\
\hline Arousals in TIB* & 6.9 & 1,8 & .031 \\
\% Awake & 13.6 & 1,8 & .006 \\
Minutes Awake & 12.7 & 1,8 & .007 \\
\% Stage 1 & 4.5 & 1,8 & .066 \\
Min Stage 1 & 5.2 & 1,8 & .053 \\
\% Stage 2 & 8.8 & 1,8 & .018 \\
Min Stage 2 & 6.7 & 1,8 & .032 \\
REMP 2nd Duration & 4.4 & 1,8 & .070 \\
REMP 2nd Min & 4.7 & 1,8 & .063 \\
REMP 3rd Duration & 3.8 & 1.8 & .086 \\
\hline
\end{tabular}

${ }^{\star} \mathrm{TIB}=$ total time in bed.

utes and percentages of each sleep stage are presented. Variables that showed significant treatment effects or trends are identified by asterisks.

Several sleep parameters showed significant changes with drug treatment. Analysis of variance summaries are presented in Table 2. Decreases in the number of arousals, in stage 1 sleep percent, and percent and

Table 1. Means and Standard Deviations of Sleep Variables

\begin{tabular}{|c|c|c|c|c|}
\hline \multirow[b]{2}{*}{ Variable } & \multicolumn{2}{|c|}{$\begin{array}{l}\text { Baseline }^{1} \\
(n=10)\end{array}$} & \multicolumn{2}{|c|}{$\begin{array}{c}\text { on Treatment } \\
(n=10)\end{array}$} \\
\hline & Mean & SD & Mean & SD \\
\hline Time in Bed (TIB) & 423.1 & $(28.6)$ & 436.8 & $(30.6)$ \\
\hline Total Sleep Period (TSP) & 401.9 & $(43.7)$ & 406.4 & $(37.0)$ \\
\hline Sleep Efficiency & 88.5 & $(5.7)$ & 90.0 & $(0.8)$ \\
\hline Sleep Latency & 11.3 & $(12.14)$ & 25.4 & $(45.5)$ \\
\hline Arousals inTIB (ARNO) & 18.2 & $(8.3)$ & $12.8^{\star}$ & $(5.2)$ \\
\hline Arousals/Last 150 Min (AR150) & 7.8 & (3.5) & 6.4 & $(4.2)$ \\
\hline REM Latency (REML) & 66.1 & $(21.2)$ & 72.1 & $(30.0)$ \\
\hline$\%$ Stage 1 & 15.1 & $(5.0)$ & $11.7^{\star \star}$ & $(3.7)$ \\
\hline$\%$ Stage 2 & 53.5 & $(8.9)$ & $58.7^{\star}$ & (7.8) \\
\hline$\%$ Stage 3 & 4.6 & $(6.2)$ & 5.4 & (8.3) \\
\hline$\%$ Stage 4 & 0.2 & $(0.5)$ & 0.2 & $(0.8)$ \\
\hline$\%$ REM & 18.8 & $(6.0)$ & 19.7 & (4.1) \\
\hline$\%$ Awake \& Movement & 7.9 & $(4.7)$ & $4.3^{\star}$ & (2.5) \\
\hline Minutes Stage 1 & 60.5 & $(20.8)$ & $47.7^{\star \star}$ & $(16.1)$ \\
\hline Minutes Stage 2 & 212.9 & $(31.2)$ & $240.2^{\star}$ & $(46.0)$ \\
\hline Minutes Stage 3 & 19.1 & $(26.1)$ & 20.5 & (31.1) \\
\hline Minutes Stage 4 & 0.7 & $(2.0)$ & 0.9 & (3.1) \\
\hline Minutes Stage REM & 76.4 & $(26.7)$ & 79.8 & $(16.5)$ \\
\hline Minutes Awake \& Movement & 32.2 & $(21.1)$ & $17.4^{\star}$ & $(10.0)$ \\
\hline REM Density & 2.1 & $(0.4)$ & 2.4 & $(0.8)$ \\
\hline 1st REMP Duration (REMDUR1) & 17.9 & (11.3) & 29.6 & $(16.4)$ \\
\hline 2nd REMP Duration (REMDUR2) & 20.7 & $(8.8)$ & $31.6^{\star \star}$ & (22.4) \\
\hline 3rd REMP Duration (REMDUR3) & 40.2 & $(25.3)$ & $32.1^{\star \star}$ & $(25.7)$ \\
\hline 1st REMP min (REMIN1) & 13.3 & $(9.4)$ & 16.7 & $(12.0)$ \\
\hline 2nd REMP min (REMIN2) & 16.0 & (7.7) & $23.3^{\star \star}$ & (17.1) \\
\hline 3rd REMP min (REMIN3) & 29.0 & (19.7) & 23.9 & $(20.0)$ \\
\hline REMP1 Density & 1.7 & $(0.6)$ & 2.1 & $(0.8)$ \\
\hline REMP2 Density & 1.9 & $(0.7)$ & 2.3 & (0.9) \\
\hline REMP3 Density & 2.0 & $(0.6)$ & 1.8 & (1.1) \\
\hline
\end{tabular}

${ }^{1}$ Average of nights 1 and 2.

${ }^{*} p<0.05$.

${ }^{\star \star} p<0.09$. 
minutes of wake and movement time were noted. Nefazodone was also associated with significant increases in the percentage and minutes of stage 2 sleep. Trends toward decreased minutes and percentage of stage 1 sleep from baseline were also obtained. The variance explained was approximately four times greater than the error term, which suggests that the effect would reach significance with a larger sample size. An increase in the duration and net REM minutes of the second REMP approached significance, whereas the length of REMP 3 appeared to decrease. Rapid-eyemovement latency showed little change from baseline, as did the minutes and percentage of stage REM.

\section{DISCUSSION}

In this acute-phase, open-trial study of nonpsychotic outpatients with major depression during treatment with nefazodone, there was a significant decrease in wakefulness and light sleep, while stage 2 sleep increased. This increased consolidation of sleep on nefazodone seemed to occur after a possible delay in sleep onset. There is also some indication that in relation to baseline, the duration and density of REM sleep may increase with treatment in the first two REMPs and decline later in the night. These effects did not reach statistical significance.

We included two duration measures describing REM episodes. One (REMDUR) reflects the total duration of the REM period, including any intervening NREM sleep or wakefulness. This statistic, compared to net REM minutes, reflects whether the REM period is consolidated or interspersed with other sleep stages. In this study, both the duration and net minutes of the first and second REM periods included during drug treatment, indicating that nefazodone may increase REM period consolidation. Further, duration and minutes of the third REM period decreased, suggesting that consolidation of REM sleep may be independent of the duration of the REM period.

Important in this study was the absence of REM suppression, a finding that differentiates nefazodone from 5-HT reuptake blockers as well as most tricyclic antidepressants and all monoamine oxidase inhibitors (Vogel et al. 1990). Unlike fluoxetine, paroxetine, and sertraline, nefazodone did not reduce REM sleep or prolong REM latency. Further, nefazodone did not increase nocturnal wakefulness and stage 1 (light) sleep, effects reported for 5-HT uptake inhibitors. Fluoxetine and paroxetine are 5-HT reuptake inhibitors, as is nefazodone (in doses over $400 \mathrm{mg} /$ day), but they lack nefazodone's effects as a $5-\mathrm{HT}_{2}$ receptor antagonist.

Interestingly, the sleep effects of nefazodone may differ substantially from those of its analogue, trazodone. Mouret et al. (1988) found that trazodone pro- longed REM latency and increased slow-wave sleep in endogenous depressed patients, without suppressing total REM sleep. On the otherhand, in a study of nonendogenous depressed outpatients who had insomnia complaints, van Bemmel et al. (1991) found that trazodone suppressed REM sleep but had no effect on deep NREM sleep. Ware et al. (1991) have also reported that trazodone suppresses REM sleep in normal controls. Although both drugs are phenylpiperazine agents, they are structurally distinct. In the present study, nefazodone did not prolong REM latency or suppress REM sleep and had no apparent effect on slow-wave sleep. These very preliminary data suggest that nefazodone and trazodone have different effects on sleep architecture.

Some investigators have suggested that at least for patients with endogenous depression, REM sleep suppression may be a predictor of antidepressant response (Kupfer et al. 1981; 1987). Vogel and colleagues have proposed that drug-induced suppression of REM sleep is, in fact, the mechanism of action of most antidepressant medications. Antidepressant-induced REM suppression may occur in both endogenous and nonendogenous depressed patients (Ware et al. 1989). Furthermore, some antidepressant drugs, such as trimipramine and nefazodone, do not suppress REM sleep, although they clearly alleviate depression. In an extensive review of drug effects on REM sleep in endogenous depression, Vogel et al. (1990) have shown 22 of 25 effective antidepressants are probable REM sleep suppressors. Trimipramine, iprindole, and amineptine did not suppress REM sleep. Thus, REM sleep suppression may be a likely mechanism of action in most antidepressants, but clearly is not the sole action of drug response.

Our results support previous studies suggesting that nefazodone has somewhat novel sleep effects. It appears to decrease light sleep as well as arousals from sleep with no decrease in total REM sleep. In fact, REM sleep periods in the first half of the night (first and second REM periods) may increase, giving way to a modest decrease in the length of the third REM period. This effect may not have reached statistical significance due to sample size.

Whether the sleep effects of nefazodone are independent of the therapeutic effects of the drug or are related to the reduction in depressive symptoms through patient-physician contact cannot be answered by these data. Comparison with studies of other antidepressants would suggest that the effects observed were caused by nefazodone rather than clinical remission alone, particularly because REM increased in the first two REM periods. Nefazodone also appears to increase REM in normal controls (Ware et al. 1991; Sharpley et al. 1992), suggesting the REM increases observed in our depressed patients are due to drug effects and not due to therapeutic response per se. Because no placebo con- 
trol was included in this study, the degree of antidepressant efficacy cannot be evaluated. However, previous clinical trials suggest that nefazodone is an effective antidepressant (Feighner et al. 1989; Fontaine et al. 1991). Furthermore, whether the sleep effects remain during prolonged nefazodone treatment is unknown. Nevertheless, although nefazodone does not suppress REM sleep, it does show promise as an antidepressant without short-term, adverse effects on sleep continuity and sleep depth. Additional research is required to evaluate the long-term sleep effects of nefazodone in a larger patient sample and to determine whether similar sleep EEG effects are found in nonresponders taking nefazodone.

\section{ACKNOWLEDGMENTS}

This study was funded by Bristol-Myers Squibb, and supported in part by a grant from The National Institute of Mental Health (MH41115). The authors wish to thank Kenneth Z. Altshuler, Chair, Department of Psychiatry for his administrative support, the technical team of the Sleep Study Unit, under the supervision of Darwynn D. Cole, B.A., for data collection and sleep-stage scoring, Doris Burton, for her secretarial support, and John Debus, M.D. for his clinical support.

\section{REFERENCES}

Armitage R, Hoffmann R, Loewy D, Moffitt A (1989): Variations in period-analyzed EEG asymmetry in REM and NREM sleep. Psychophysiology 26:329-336.

Delgado PL, Price L, Miller HL, Salomon RM, Licinio J, Krystal JH, Heninger GR, Charney BJ (1991): Rapid serotonin depletion as a provocativechallenge test for patients with major depression: Relevance to antidepressant action and the neurobiology of depression. Psychopharmacol Bull 27:321-330

Eison AS, Eison MS, Torrente JR, Wright RN, Yocca FD (1990): Nefazodone: Preclinical pharmacology of a new antidepressant. Psychopharmacol Bull 26:311-315.

Feighner JP, Pambakian R, Fowler RC, Boyler WF, D'Amico MF (1989): A comparison of nefazodone, imipramine and placebo in patients with moderate to severe depression. Psychopharmacol Bull 25:219-221

Fontaine R, Ontiveros A, Faludi G, Elie R, Roberts D, Ecker J (1991): A study of nefazodone, imipramine and placebo in depressed outpatients. Biol Psychiatry 29:364S (Abstract)

Hamilton M (1960): A rating scale for depression. J Neurol Neurosurg Psychiatry 23:56-62

Kay DC, Blackburn AB, Buckingham JA, Karacan I (1976): Human pharmacology of sleep. In Williams RL, Karacan I(eds), Pharmacology of Sleep, New York, JohnWiley \& Sons, pp 83-210

Kupfer DJ, Foster GF, Reid L, Thompson KS, Weiss B (1987):
EEG sleep changes as predictors in depression. Am J Psychiatry 133:622-626

KupferDJ, Spiker DG, Coble PA, Neil JF, Ulrich R, Shaw DH (1981): Sleep and treatment prediction in endogenous depression. Am J Psychiatry 138:429-433

Jones BE (1991): Paradoxical sleep and its chemical/structural substrates in the brain. Neuroscience 40:637-656

Mouret J, Lemoine P, Minuit MP, Benkelfat C, Renardet M (1988): Effects of trazodone on the sleep of depressed subjects, a polysomnographic study. Psychopharmacology 95:537-543

Rechtschaffen A, Kales A (1968): A Manual of Standardized Terminology, Techniques and Scoring System for Sleep Stages of Human Subjects, Los Angeles, Brain Information Service, Brain Research Institute, University of California

Rush AJ, Giles DE, Jarrett RJ, Feldman-Koffler F, Debus JR, Weissenburger JE, Orsulak PJ, Roffwarg HP (1989): Reduced REM latency predicts response to tricyclic medication in depressed outpatients. Biol Psychiatry 26:61-72

Rush AJ, Cain JW, Reese J, Stewart RS, Waller DA, Debus JD (1991): Neurological bases for psychiatric disorders. In Rosenberg RN (ed), Comprehensive Neurology, New York, Raven Press, pp 555-603

Saletu B, Frey R, Krupka M, Anderer P, Grunberger J, See WR (1991): Sleep laboratory studies on the single-dose effects of serotonin reuptake inhibitors paroxetine and fluoxetine on human sleep and awakening qualities. Sleep 14:439-447

Sharpley AL, Walsh AES, Cowen PJ (1992): Nefazodone-a novel antidepressant - may increase REM sleep. Biol Psychiatry 31:1070-1073

Siegel JM (1990): Mechanisms of sleep control. J Clin Neurophysiol 7:49-65

Spitzer RL, Williams JBW, Gibbons M (1986): The Structured Clinical Interview for DSM-III-R (SCID), New York, New York State Psychiatric Research Institute

Stevens J (1986): Applied Multivariate Statistics for the Social Sciences, Hillsdale, Lawrence Erlbaum Associates

van Bemmel AL, Haversman RG, van Diest R (1991): Effects of trazodone on sleep and clinical state in major depression. Psychopharmacology 107:569-574

Vogel GW, Thompson Jr FC, Thurmond A, Giesler D, Barrowclough $B$ (1972): The effect of REM deprivation on depressive syndromes. Sleep Res 1:167 (Abstract)

Vogel GW (1983): Evidence for REM sleep deprivation as the mechanism of action of antidepressant drugs. Prog Neuropharmacol Biol Psychiatry 7:343-349

Vogel GW, Buffenstein A, Minter K, Hennessey A (1990): Drug effects on REM sleep and on endogenous depression. Neurosci Biobehav Rev 14:49-63

Ware JC, Brown FW, Moorad Jr PJ, Pittard JT, Cobert B (1989): Effects on sleep: A double-blind study comparing trimipramine to imipramine in depressed insomniac patients. Sleep 12(6):537-549

Ware JC, Rose V, McBrayer R (1991): The effects of nefazodone, trazodone, buspirone and placebo on sleep and sleep related penile erections (NPT) in normal subjects. Sleep Res 20:91 